\title{
A sequential trial of tolbutamide in hypoproteinaemia in adults
}

\author{
G. S. MUTALIK AND INDER SINGH
}

From the Department of Medicine, B. J. Medical College, Poona, India

EDITORIAL SYNOPSIS Tolbutamide, $1.0 \mathrm{~g}$. daily, has been shown to cause clinical improvement in patients with malnutrition and was associated with an increase in plasma proteins.

A definite increase in the glycogen content of the liver with tolbutamide administration in animal experiments has prompted some workers to investigate the action of the drug on liver diseases in man. Such an idea was based on the assumption that augmenting glycogen in the liver must have a beneficial effect on the damaged liver. Knick and Emrich (1957) used tolbutamide, $3 \mathrm{~g}$. daily for three to six weeks, in six cases of chronic hepatitis and six cases of liver cirrhosis without diabetes and reported increased appetite, decrease in serum bilirubin, marked restoration to normal of the serum proteins, and improvement in the bromsulphalein test. Improvement in bromsulphalein excretion was also reported in a diabetic patient by Pote, Anderson, and Cochran (1959). László, Bruckner, Görgey, and Tóth (1959) treated 50 patients suffering from acute hepatitis and found that decrease in serum bilirubin and recovery time were both hastened by tolbutamide when compared with a similar group of controls. In liver biopsy studies Dobrzański (1959) noted excess of glycogen in liver cells, diminution of vacuolization of liver cell nuclei, and distinct regression of fatty changes in the liver in 12 tolbutamide-treated diabetic patients.

Singh, Sehra, and Bhargava (1961) treated 55 cases of cirrhosis of the liver suffering from anorexia, emaciation, anaemia, hypoproteinaemia, and gross ascites with tolbutamide and reported improvement in appetite in 45 cases $(82 \%)$, control of ascites in 42 cases $(76 \%)$, weight gain in 36 cases $(66 \%)$, increase in serum proteins in 35 cases $(64 \%)$, and improvement in the haemogram in 24 cases (44\%). Liver-function tests showed moderate improvement in 26 cases $(47 \%)$ but there was no deterioration in the remaining 29 cases. Bhattacharyya and Mandal (1963) reported comparable results in 10 cases of cirrhosis of the liver from the Calcutta School of Tropical Medicine. Under tolbutamide therapy total serum proteins improved progressively in seven cases, there was initial improvement followed by deterioration in two cases, and practically no change in one case. Rapid improvement in the albumin fraction was a striking feature when the initial serum albumin level was below $2 \mathrm{~g} . \%$.

It was felt by us that the increase in serum proteins under tolbutamide administration may not necessarily be confined to diseased livers. Thus, pretreatment of rats with tolbutamide had been observed to increase the incorporation of ${ }^{14} \mathrm{C}$-glycine into protein in liver slices by Recant and Fischer (1957); and DeMeutter, Khachadurian, and Marble (1958) had noticed that tolbutamide injected intravenously into human subjects decreased serum $\alpha$-amino-acids. We were, therefore, prompted to try the effect of tolbutamide in the treatment of hypoproteinaemia from any cause. Using the restricted closed design sequential trial technique, advocated by Armitage (1960), we have found the results to be satisfying in malnutrition and malabsorption; previous reports of increase in serum proteins with tolbutamide in cirrhosis of the liver (vide supra) have been confirmed.

MATERIAL AND METHOD

The patients were 40 Indians, 28 men and 12 women, 24 to 60 years old. They were admitted to hospital for debility, oedema, and hypoproteinaemia.

An initial estimate of the clinical status of each patient (vide infra) was made and the cause of his hypoproteinaemia was determined. Those resulting from malnutrition, malabsorption, and cirrhosis of the liver were selected for the trial; cases with malignant disease and tuberculosis of the bowel were not included.

During the period of investigation of the cause of hypoproteinaemia the opportunity was taken to accustom the patients to their environment. When the investigations permitted they were given the standard high-protein 
hospital diet and tablet B (placebo) twice daily before food.

These investigations were completed in two weeks and the patients were then ready for the tolbutamide trial. A pre-treatment estimate of the clinical status of each patient, comprising his sense of well-being, state of oedema, weight, and the serum proteins, total as well as albumin and globulin, was made. The patients were weighed to the nearest $0.05 \mathrm{~kg}$. and the proteins were estimated by the biuret method as described by Harrison (1957). The trial, which was double blind, then commenced. All comparable cases were paired by randomization. In each pair one patient received tablet $A$ (tolbutamide) and the other tablet B (placebo) twice daily before food. The dose of tolbutamide was $1.0 \mathrm{~g}$. daily. All patients continued to remain on the standard high-protein hospital diet to which they had been introduced earlier. The therapy was continued for four weeks and the post-treatment estimate of the clinical status and of the serum proteins was made in each case on its termination.

Results were assessed by sequential analysis. The criteria for assessment, whether in each group therapy A or B was better of the two, were (1) subjective improvement, (2) weight gain, (3) reduction in oedema, and (4) improvement in serum protein levels. One treatment was regarded as being superior to the other which was superior with at least three out of the four assessments. The pairs were thus designated $\mathrm{AB}$ ( $\mathrm{A}$ better than $\mathrm{B}$ ), BA (B better than A), or T (tied pair in which a distinction could not be made between the two drugs).

A restricted closed design was chosen for the experiment. Before the trial began it was estimated that a placebo was unlikely to produce noticeable improvement, as judged by the criteria, in more than $15 \%$ of the cases. If tolbutamide raised this by $50 \%$, i.e., to $65 \%$, then it could be claimed that the difference was sufficiently great in favour of the drug. From this combination, $\pi_{1}=0.65$ and $\pi_{2}=0.15$, the value of $\theta_{1}=0.913$ (approximately 0.9) was obtained (Table $4 \cdot 1$ of Armitage, 1960). The boundaries were drawn with $\theta_{1}=0.9,2 \alpha=0.05$, and $1-\beta=0.95$ (Table 3.7 of Armitage, 1960).

\section{RESULTS}

Table I gives the experimental data. The causes of hypoproteinaemia were malnutrition (12 cases), malabsorption (20 cases), and cirrhosis of the liver (eight cases). The pre-treatment protein levels varied from $2 \cdot 2$ to $4.8 \mathrm{~g}$. \%. Forty patients (20 pairs) entered the trial. On the basis of criteria for assessment, two pairs were tied and 18 pairs were untied, 15 pairs for tolbutamide and three pairs for placebo.

Figure 1 shows the experiment. The sample path is drawn with the number of pairs against the excess preferences. The experiment was concluded at the eighteenth pair when the upper boundary was crossed by the path and a significant advantage for tolbutamide therapy was established.

\section{DISCUSSION}

In Table I and Fig. 1 there is a definite advantage to group A (tolbutamide therapy). The value of the treatment has been enhanced by collation of evidence from all four criteria of assessment, i.e., subjective improvement, weight gain, reduction of oedema, and improvement in serum protein levels into a single judgment whether tolbutamide was a success against placebo controls or not, and by limiting the duration of the trial to four weeks. Thus, discrepancies in assessment of results due to chance, inaccuracy in protein estimations, or loss of objectivity of any single parameter have been avoided. Table II gives details about the changes which took place in each patient between admission to the hospital and entry to the trial. There is no indication in Table II that, by at least three criteria of assessment, group A cases were by any chance doing better before the treatment started. The rises noted in serum proteins in some group A or group B cases are too insignificant for consideration.

We are not sure about the exact mode of action of tolbutamide in these cases. It appears, however, that the beneficial effect may accrue from (1) insulin release, (2) increased sodium excretion, and (3) improved digestion.

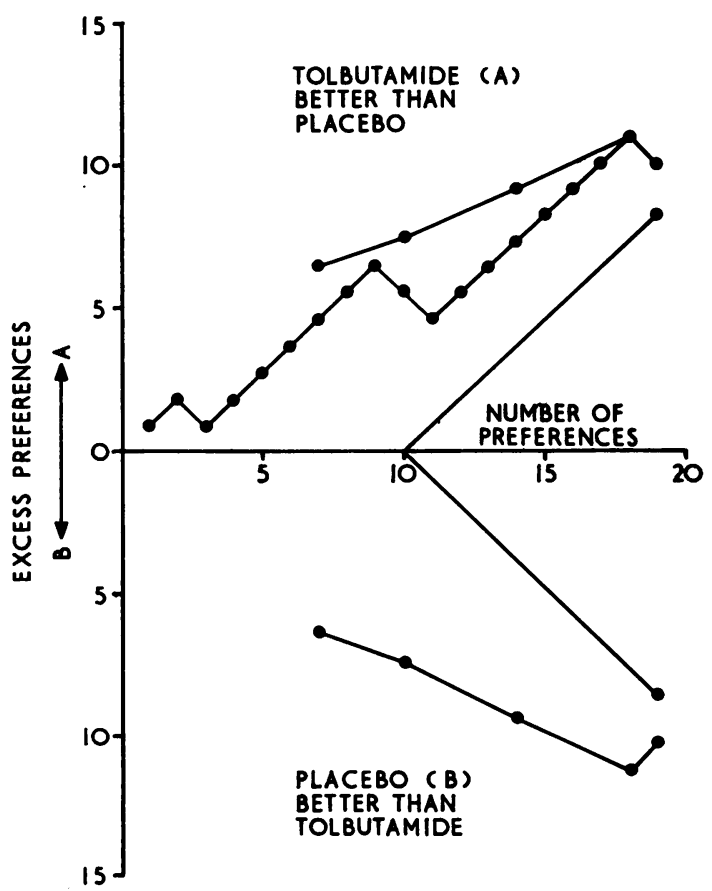

FIG. 1. Treatment with tolbutamide versus placebo. Restricted closed design with $2 \alpha=0.05,1-\beta=0.95, \theta$ $=0.90$, and $N=19$. 
TABLE I

Pair No. Age Sex Cause of $\begin{aligned} & \text { Pair No. Age Sex } \\ & \text { and } \\ & \text { Treatment } \\ & \text { Group }\end{aligned}$
$\begin{aligned} & \text { Cause of } \\ & \text { Hypoproteinaemia }\end{aligned}$ EXPERIMENTAL DATA (AFTER TREATMENT)

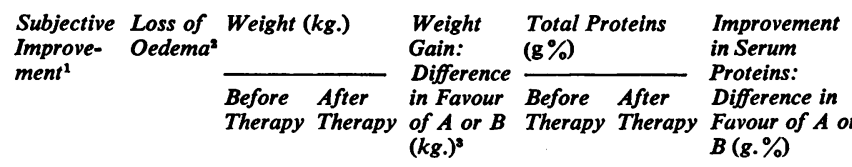
$\begin{array}{llll}\begin{array}{l}\text { Gain: } \\ \text { Difference }\end{array} & (\mathrm{g} \%) & \begin{array}{l}\text { In Serum } \\ \text { Proteins: }\end{array} \\ \begin{array}{l}\text { Before After in Favour Before After } \\ \text { Difference in } \\ \text { Therapy Therapy of A or B }\end{array} & \text { Therapy Therapy Favour of A or }\end{array}$ $(\mathrm{kg} .)^{3}$

Advantages to Typesce
Aor Bas adr
Judged by the
Criteria

\begin{tabular}{|c|c|c|c|c|c|c|c|c|c|c|c|c|c|c|}
\hline 1 & $\mathbf{A}$ & 38 & $\mathbf{M}$ & Protein malnutrition & Good & ++ & $62 \cdot 85$ & $64 \cdot 30$ & $\begin{array}{l}0.40 \\
\mathbf{A}\end{array}$ & $3 \cdot 8$ & $4 \cdot 8$ & $\begin{array}{l}0.8 \\
\mathbf{A}\end{array}$ & $\mathbf{A}$ & $\mathbf{A B}$ \\
\hline & B & 42 & $\mathbf{M}$ & Protein malnutrition & Poor & - & $68 \cdot 45$ & 69.50 & & 2.8 & 3.0 & & & \\
\hline 2 & $\mathbf{A}$ & 58 & $\mathbf{M}$ & Malabsorption syndrome & Good & +++ & $56 \cdot 50$ & $58 \cdot 00$ & $\begin{array}{l}0.35 \\
\mathbf{A}\end{array}$ & $3 \cdot 6$ & $4 \cdot 2$ & $\begin{array}{l}0 \cdot 6 \\
\text { A }\end{array}$ & $\mathbf{A}$ & $\mathbf{A B}$ \\
\hline & B & 50 & $\mathbf{M}$ & Malabsorption syndrome & Poor & + & $60 \cdot 10$ & $61 \cdot 25$ & & $3 \cdot 8$ & $3 \cdot 8$ & & & \\
\hline 3 & $\mathbf{A}$ & 48 & $\mathbf{F}$ & Malabsorption syndrome & Moderate & ++ & 58.85 & $60 \cdot 35$ & $\begin{array}{l}0.55 \\
B\end{array}$ & $3 \cdot 4$ & $4 \cdot 0$ & $\begin{array}{l}0.4 \\
\text { B }\end{array}$ & $\mathbf{B}$ & BA \\
\hline & B & 40 & $\mathbf{F}$ & Malabsorption syndrome & Moderate & ++ & $54 \cdot 60$ & 56.65 & & $3 \cdot 2$ & $4 \cdot 2$ & & & \\
\hline 4 & $\mathbf{A}$ & 60 & $\mathbf{F}$ & Malnutrition & Moderate & ++ & $60 \cdot 45$ & $62 \cdot 30$ & $\begin{array}{l}0.55 \\
\mathrm{~A}\end{array}$ & $4 \cdot 0$ & $5 \cdot 4$ & $1 \cdot 0$ & $\mathbf{A}$ & $\mathbf{A B}$ \\
\hline & $\mathbf{B}$ & 48 & $\mathbf{F}$ & Malnutrition & Poor & - & 55.05 & $56 \cdot 35$ & & $3 \cdot 8$ & $4 \cdot 2$ & & & \\
\hline 5 & $\mathbf{A}$ & 42 & $\mathbf{M}$ & Malabsorption & Good & +++ & $64 \cdot 25$ & 65.85 & $\begin{array}{l}0.40 \\
\text { A }\end{array}$ & $4 \cdot 0$ & $5 \cdot 8$ & $\begin{array}{l}1.5 \\
\mathrm{~A}\end{array}$ & $\mathbf{A}$ & $\mathbf{A B}$ \\
\hline & B & 38 & $\mathbf{M}$ & Malabsorption & Poor & - & $71 \cdot 25$ & $72 \cdot 45$ & & $4 \cdot 1$ & $4 \cdot 4$ & & & \\
\hline 6 & $\mathbf{A}$ & 50 & $\mathbf{M}$ & Cirrhosis of liver & Moderate & ++ & 59.90 & $60 \cdot 10$ & $\begin{array}{l}0.25 \\
\mathrm{~A}\end{array}$ & $3 \cdot 0$ & $4 \cdot 6$ & $\begin{array}{l}1.8 \\
A\end{array}$ & $\mathbf{A}$ & $\mathbf{A B}$ \\
\hline & B & 46 & $\mathbf{M}$ & Cirrhosis of liver & Poor & - & 55.90 & $55 \cdot 85$ & & $3 \cdot 2$ & $3 \cdot 0$ & & & \\
\hline 7 & $\mathbf{A}$ & 45 & $\mathbf{M}$ & Malnutrition & Good & +++ & $67 \cdot 20$ & $69 \cdot 35$ & $\begin{array}{l}0.65 \\
\text { A }\end{array}$ & $3 \cdot 6$ & $5 \cdot 4$ & $\begin{array}{l}1.4 \\
\text { A }\end{array}$ & $\mathbf{A}$ & $\mathbf{A B}$ \\
\hline & $\mathbf{B}$ & 38 & $\mathbf{M}$ & Malnutrition & Moderate & + & $63 \cdot 35$ & $64 \cdot 85$ & & $2 \cdot 8$ & $3 \cdot 2$ & & & \\
\hline 8 & $\mathbf{A}$ & 43 & $\mathbf{F}$ & Chronic diarrhoea & Good & ++ & $58 \cdot 40$ & $60 \cdot 40$ & $\begin{array}{l}0.65 \\
\mathrm{~A}\end{array}$ & $4 \cdot 0$ & $6 \cdot 0$ & $\begin{array}{l}1 \cdot 8 \\
A\end{array}$ & $\mathbf{A}$ & $\mathbf{A B}$ \\
\hline & $\mathbf{B}$ & 48 & $\mathbf{F}$ & Chronic diarrhoea & Poor & - & $51 \cdot 85$ & $53 \cdot 20$ & & $3 \cdot 6$ & $3 \cdot 8$ & & & \\
\hline 9 & $\mathbf{A}$ & 52 & $\mathbf{M}$ & Post-dysenteric syndrome & Good & ++ & $65 \cdot 60$ & $66 \cdot 80$ & $\begin{array}{l}0.45 \\
\mathrm{~A}\end{array}$ & $3 \cdot 2$ & $6 \cdot 4$ & $\begin{array}{l}2 \cdot 4 \\
\text { A }\end{array}$ & $\mathbf{A}$ & $\mathbf{A B}$ \\
\hline & $\mathbf{B}$ & 58 & $\mathbf{M}$ & Chronic diarrhoea & Moderate & + & $68 \cdot 70$ & $69 \cdot 45$ & & $2 \cdot 8$ & $3 \cdot 6$ & & & \\
\hline 10 & $\mathbf{A}$ & 38 & $\mathbf{F}$ & Malabsorption & Moderate & + & $52 \cdot 60$ & $52 \cdot 55$ & $\begin{array}{l}0.35 \\
\text { B }\end{array}$ & $3 \cdot 2$ & 3.4 & $\begin{array}{l}1 \cdot 6 \\
B\end{array}$ & B & BA \\
\hline & B & 34 & $\mathbf{F}$ & Malabsorption & Good & ++ & $56 \cdot 35$ & $56 \cdot 65$ & & $3 \cdot 0$ & $4 \cdot 8$ & & & \\
\hline 11 & $\mathbf{A}$ & 54 & $\mathbf{M}$ & Malnutrition & Poor & ++ & 58.65 & 58.75 & $\begin{array}{l}0 \cdot 10 \\
A\end{array}$ & $2 \cdot 8$ & 3.4 & $\begin{array}{l}0 \cdot 1 \\
\mathrm{~A}\end{array}$ & $\begin{array}{l}\text { No advantage } \\
\text { to either }\end{array}$ & $\mathbf{T}$ \\
\hline & $\mathbf{B}$ & 60 & $\mathbf{M}$ & Malnutrition & Moderate & ++ & $64 \cdot 40$ & $64 \cdot 40$ & & $3 \cdot 0$ & $3 \cdot 5$ & & & \\
\hline 12 & $\mathbf{A}$ & 56 & $\mathbf{M}$ & Cirrhosis of liver & Poor & + & $64 \cdot 30$ & 64.45 & $\begin{array}{l}0.35 \\
\text { B }\end{array}$ & $4 \cdot 8$ & 5.0 & $\begin{array}{l}1 \cdot 0 \\
B\end{array}$ & $\mathbf{B}$ & BA \\
\hline & B & 50 & $\mathbf{M}$ & Cirrhosis of liver & Moderate & ++ & $60 \cdot 25$ & $60 \cdot 75$ & & $4 \cdot 6$ & 5.8 & & & \\
\hline 13 & $\mathbf{A}$ & 28 & $\mathbf{F}$ & Malnutrition & Moderate & + & 42.45 & 43.65 & $\begin{array}{l}0.40 \\
\text { A }\end{array}$ & $4 \cdot 4$ & 5.4 & $\begin{array}{l}0.6 \\
\text { A }\end{array}$ & $\mathbf{A}$ & $\mathbf{A B}$ \\
\hline & B & 24 & $\mathbf{F}$ & Malnutrition & Poor & - & $45 \cdot 30$ & $46 \cdot 10$ & & $4 \cdot 2$ & $4 \cdot 6$ & & & \\
\hline 14 & $\mathbf{A}$ & 50 & $\mathbf{M}$ & Cirrhosis of liver & Good & ++ & $68 \cdot 25$ & $69 \cdot 45$ & $\begin{array}{l}0.60 \\
\text { A }\end{array}$ & 4.0 & 5.0 & $\begin{array}{l}0.7 \\
\text { A }\end{array}$ & $\mathbf{A}$ & $\mathbf{A B}$ \\
\hline & B & 40 & $\mathbf{M}$ & Cirrhosis of liver & Poor & - & $73 \cdot 40$ & 74.00 & & 3.8 & $4 \cdot 1$ & & & \\
\hline 15 & $\mathbf{A}$ & 48 & $\mathbf{M}$ & Malabsorption & Good & ++ & $51 \cdot 15$ & 52.45 & $\begin{array}{l}0.55 \\
\mathrm{~A}\end{array}$ & 3.8 & $4 \cdot 6$ & $\begin{array}{l}0 \cdot 6 \\
\text { A }\end{array}$ & $\mathbf{A}$ & $\mathbf{A B}$ \\
\hline & B & 43 & $\mathbf{M}$ & Malabsorption & Moderate & - & $58 \cdot 30$ & 59.05 & & $3 \cdot 6$ & $3 \cdot 8$ & & & \\
\hline 16 & $\mathbf{A}$ & 57 & $\mathbf{M}$ & Malabsorption & Moderate & + & $65 \cdot 30$ & $65 \cdot 35$ & $\begin{array}{l}\mathbf{0} \cdot 10 \\
\mathrm{~B}\end{array}$ & $4 \cdot 0$ & $4 \cdot 2$ & $\begin{array}{l}0.2 \\
\mathbf{A}\end{array}$ & $\begin{array}{l}\text { No advantage } \\
\text { to either }\end{array}$ & $\mathbf{T}$ \\
\hline & B & 49 & $\mathbf{M}$ & Malabsorption & Moderate & ++ & $70 \cdot 70$ & $70 \cdot 85$ & & 3.8 & $3 \cdot 8$ & & & \\
\hline 17 & $\mathbf{A}$ & 40 & $\mathbf{M}$ & Malabsorption & Good & ++ & $72 \cdot 55$ & $73 \cdot 85$ & $\begin{array}{l}0.40 \\
\mathrm{~A}\end{array}$ & $4 \cdot 2$ & $5 \cdot 4$ & $\begin{array}{l}0 \cdot 6 \\
\text { A }\end{array}$ & $\mathbf{A}$ & $\mathbf{A B}$ \\
\hline & B & 46 & $\mathbf{M}$ & Malabsorption & Poor & - & $64 \cdot 75$ & $65 \cdot 65$ & & $4 \cdot 0$ & $4 \cdot 6$ & & & \\
\hline 18 & $\mathbf{A}$ & 42 & $\mathbf{F}$ & Cirrhosis of liver & Good & +++ & $58 \cdot 20$ & $59 \cdot 35$ & $\begin{array}{l}0.50 \\
\mathbf{A}\end{array}$ & $3 \cdot 2$ & 5.0 & $\begin{array}{l}1.2 \\
\text { A }\end{array}$ & $\mathbf{A}$ & $\mathbf{A B}$ \\
\hline & B & 43 & $\mathbf{M}$ & Cirrhosis of liver & Poor & - & $54 \cdot 85$ & $55 \cdot 50$ & & $3 \cdot 0$ & 3.6 & & & \\
\hline 19 & $\mathbf{A}$ & 24 & $\mathbf{M}$ & Malnutrition & Moderate & ++ & $50 \cdot 25$ & 51.00 & $\begin{array}{l}0.40 \\
\text { A }\end{array}$ & $4 \cdot 2$ & $5 \cdot 4$ & $\begin{array}{l}1.4 \\
\text { A }\end{array}$ & $\mathbf{A}$ & $\mathbf{A B}$ \\
\hline & B & 36 & $\mathbf{M}$ & Malnutrition & Poor & - & 52.65 & 53.00 & & $3 \cdot 8$ & $3 \cdot 6$ & & & \\
\hline 20 & $\mathbf{A}$ & 40 & $\mathbf{M}$ & Malabsorption & Good & ++ & 56.05 & $57 \cdot 30$ & $\begin{array}{l}0.30 \\
\text { A }\end{array}$ & $2 \cdot 2$ & $3 \cdot 6$ & $\begin{array}{l}0 \cdot 6 \\
\text { A }\end{array}$ & $\mathbf{A}$ & $\mathbf{A B}$ \\
\hline & B & 48 & $\mathbf{F}$ & Malabsorption & Poor & - & $54 \cdot 25$ & $55 \cdot 20$ & & $2 \cdot 8$ & $3 \cdot 6$ & & & \\
\hline
\end{tabular}


TABLE II

EXPERIMENTAL DATA (BEFORE TREATMENT)

Pair No. Age Sex Cause of

and
Treatment

\section{Group}

\begin{tabular}{|c|c|c|c|c|c|c|c|c|c|}
\hline \multirow{2}{*}{$\begin{array}{l}\text { Subjective } \\
\text { Improve- } \\
\text { ment }^{1}\end{array}$} & \multirow{2}{*}{$\begin{array}{l}\text { Loss of } \\
\text { Oedema }^{2}\end{array}$} & \multicolumn{2}{|c|}{ Weight (kg.) } & \multirow{2}{*}{$\begin{array}{l}\text { Weight } \\
\text { Gain: } \\
\text { Difference } \\
\text { in Favour } \\
\text { of A or } \\
\text { B (kg.) }\end{array}$} & \multicolumn{2}{|c|}{$\begin{array}{l}\text { Total Proteins } \\
(\mathrm{g} . \%)\end{array}$} & \multirow{2}{*}{$\begin{array}{l}\text { Improvement } \\
\text { in Serum } \\
\text { Proteins: } \\
\text { Difference in } \\
\text { Favour of } \\
\text { A or B }(\mathrm{g} . \%)\end{array}$} & \multirow{2}{*}{$\begin{array}{l}\text { Advantages } \\
\text { to } A \text { or } B \\
\text { as Judged } \\
\text { by Criteria }\end{array}$} & \multirow{2}{*}{$\begin{array}{l}\text { Types of } \\
\text { Pair }\end{array}$} \\
\hline & & $\begin{array}{l}\text { On } \\
\text { Admission }\end{array}$ & $\begin{array}{l}\text { Before } \\
\text { Therapy }\end{array}$ & & $\begin{array}{l}\text { On } \\
\text { Admission }\end{array}$ & $\begin{array}{l}\text { Before } \\
\text { Therapy }\end{array}$ & & & \\
\hline
\end{tabular}
$B$ (kg.)

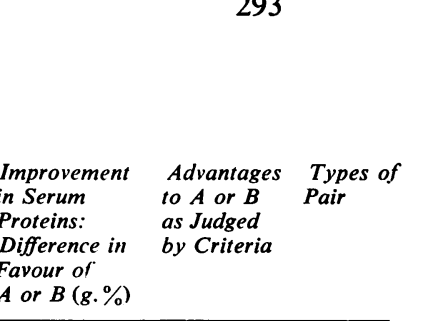

की

\begin{tabular}{|c|c|c|c|c|c|c|c|c|c|c|c|c|c|c|}
\hline \multirow[t]{2}{*}{1} & A & 38 & $\mathbf{M}$ & Protein malnutrition & Nil & Nil & 62.95 & $62 \cdot 85$ & $0 \cdot 20$ & $3 \cdot 8$ & $3 \cdot 8$ & $\begin{array}{l}0 \cdot 2 \\
\mathrm{~B}\end{array}$ & To none & $\mathrm{T}$ \\
\hline & B & 42 & $\mathbf{M}$ & Protein malnutrition & Nil & Nil & $68 \cdot 35$ & $68 \cdot 45$ & & $2 \cdot 6$ & $2 \cdot 8$ & & & \\
\hline \multirow[b]{2}{*}{2} & A & 58 & $\mathbf{M}$ & Malabsorption syndrome & Nil & Nil & $56 \cdot 50$ & $56 \cdot 50$ & $0 \cdot 10$ & $3 \cdot 6$ & $3 \cdot 6$ & Nil & To none & $\mathbf{T}$ \\
\hline & B & 50 & $\mathbf{M}$ & Malabsorption syndrome & Nil & Nil & $60 \cdot 00$ & $60 \cdot 10$ & B & $3 \cdot 8$ & $3 \cdot 8$ & & & \\
\hline \multirow{2}{*}{3} & $\mathbf{A}$ & 48 & $F$ & Malabsorption syndrome & Nil & Nil & 59.00 & $58 \cdot 85$ & $0 \cdot 30$ & 3.4 & $3 \cdot 4$ & $0 \cdot 1$ & To none & $\mathbf{T}$ \\
\hline & B & 40 & $\mathrm{~F}$ & Malabsorption syndrome & Nil & Nil & 54.45 & $54 \cdot 60$ & & $3 \cdot 3$ & $3 \cdot 2$ & & & \\
\hline \multirow{2}{*}{4} & $\mathbf{A}$ & 60 & $\mathrm{~F}$ & Malnutrition & Nil & Nil & $60 \cdot 30$ & $60 \cdot 45$ & $0 \cdot 10$ & $3 \cdot 8$ & $4 \cdot 0$ & $0 \cdot 1$ & To none & $\mathbf{T}$ \\
\hline & B & 48 & $\mathbf{F}$ & Malnutrition & Nil & Nil & 55.00 & 55.05 & & 3.5 & 3.8 & & & \\
\hline \multirow[b]{2}{*}{5} & $\mathbf{A}$ & 50 & $\mathbf{M}$ & Malabsorption & Nil & Nil & $64 \cdot 15$ & $64 \cdot 25$ & 0.05 & $4 \cdot 1$ & $4 \cdot 0$ & $0 \cdot 3$ & To none & $\mathbf{T}$ \\
\hline & B & 38 & $\mathbf{M}$ & Malabsorption & Nil & Nil & $71 \cdot 10$ & $71 \cdot 25$ & B & 3.9 & $4 \cdot 1$ & B & & \\
\hline \multirow{2}{*}{6} & $\mathbf{A}$ & 50 & $\mathbf{M}$ & Cirrhosis of liver & Nil & Nil & $60 \cdot 00$ & $59 \cdot 90$ & $0 \cdot 10$ & $2 \cdot 9$ & $3 \cdot 0$ & Nil & To none & $\mathbf{T}$ \\
\hline & B & 46 & $\mathbf{M}$ & Cirrhosis of liver & Nil & Nil & $56 \cdot 10$ & $55 \cdot 90$ & & $3 \cdot 1$ & $3 \cdot 2$ & & & \\
\hline \multirow{2}{*}{7} & $\mathbf{A}$ & 45 & $\mathbf{M}$ & Malnutrition & Nil & Nil & $67 \cdot 20$ & $67 \cdot 20$ & $0 \cdot 20$ & $3 \cdot 6$ & $3 \cdot 6$ & $0 \cdot 1$ & To none & $\mathbf{T}$ \\
\hline & B & 38 & $\mathbf{M}$ & Malnutrition & Nil & Nil & $63 \cdot 15$ & $63 \cdot 35$ & & $2 \cdot 7$ & $2 \cdot 8$ & & & \\
\hline \multirow{2}{*}{8} & $\mathbf{A}$ & 43 & $\mathrm{~F}$ & Chronic diarrhoea & Nil & Nil & $58 \cdot 20$ & 58.40 & Nil & $3 \cdot 6$ & $4 \cdot 0$ & $0 \cdot 2$ & To none & $\mathbf{T}$ \\
\hline & B & 48 & $\mathrm{~F}$ & Chronic diarrhoea & Nil & Nil & $51 \cdot 65$ & $51 \cdot 85$ & & $3 \cdot 4$ & $3 \cdot 6$ & & & \\
\hline \multirow{2}{*}{9} & $\mathbf{A}$ & 52 & $\mathbf{M}$ & Post-dysenteric syndrome & Nil & Nil & $65 \cdot 80$ & $65 \cdot 60$ & $0 \cdot 30$ & $2 \cdot 8$ & $3 \cdot 2$ & Nil & To none & $\mathbf{T}$ \\
\hline & B & 58 & $\mathbf{M}$ & Chronic diarrhoea & Nil & Nil & $68 \cdot 60$ & $68 \cdot 70$ & & $2 \cdot 4$ & $2 \cdot 8$ & & & \\
\hline \multirow{2}{*}{10} & $\mathbf{A}$ & 38 & $\mathbf{F}$ & Malabsorption & Nil & Nil & $52 \cdot 60$ & $52 \cdot 60$ & 0.05 & $3 \cdot 0$ & $3 \cdot 2$ & $0 \cdot 2$ & To none & $\mathbf{T}$ \\
\hline & B & 34 & $\mathrm{~F}$ & Malabsorption & Nil & Nil & $56 \cdot 40$ & $56 \cdot 35$ & & $3 \cdot 0$ & $3 \cdot 0$ & & & \\
\hline \multirow{2}{*}{11} & $\mathbf{A}$ & 54 & $\mathbf{M}$ & Malnutrition & Nil & Nil & $58 \cdot 50$ & 58.65 & $0 \cdot 10$ & $2 \cdot 8$ & $2 \cdot 8$ & Nil & To none & $\mathbf{T}$ \\
\hline & B & 60 & $\mathbf{M}$ & Malnutrition & Nil & Nil & $64 \cdot 35$ & $64 \cdot 40$ & & 3.0 & $3 \cdot 0$ & & & \\
\hline \multirow[b]{2}{*}{12} & $\mathbf{A}$ & 56 & $\mathbf{M}$ & Cirrhosis of liver & Nil & Nil & $64 \cdot 25$ & $64 \cdot 30$ & 0.05 & $4 \cdot 6$ & $4 \cdot 8$ & $0 \cdot 1$ & To none & $\mathbf{T}$ \\
\hline & B & 50 & $\mathbf{M}$ & Cirrhosis of liver & Nil & Nil & $60 \cdot 15$ & $60 \cdot 25$ & B & $4 \cdot 5$ & $4 \cdot 6$ & A & & \\
\hline \multirow[b]{2}{*}{13} & $\mathbf{A}$ & 28 & $\mathbf{F}$ & Malnutrition & Nil & Nil & $42 \cdot 25$ & $42 \cdot 45$ & $0 \cdot 10$ & $4 \cdot 3$ & $4 \cdot 4$ & $0 \cdot 1$ & To none & $\mathbf{T}$ \\
\hline & B & 24 & $\mathrm{~F}$ & Malnutrition & Nil & Nil & 45.00 & $45 \cdot 30$ & B & $4 \cdot 0$ & $4 \cdot 2$ & B & & \\
\hline \multirow{2}{*}{14} & A & 50 & $\mathbf{M}$ & Cirrhosis of liver & Nil & Nil & 68.05 & $68 \cdot 25$ & Nil & $3 \cdot 8$ & $4 \cdot 0$ & $0 \cdot 1$ & To none & $\mathbf{T}$ \\
\hline & B & 40 & $\mathbf{M}$ & Cirrhosis of liver & Nil & Nil & $73 \cdot 20$ & $73 \cdot 40$ & & $3 \cdot 5$ & $3 \cdot 8$ & & & \\
\hline \multirow{2}{*}{15} & $\mathbf{A}$ & 48 & $\mathbf{M}$ & Malabsorption & Nil & $\mathrm{Nil}$ & $50 \cdot 85$ & $51 \cdot 15$ & 0.05 & $3 \cdot 7$ & $3 \cdot 8$ & $0 \cdot 1$ & To none & $\mathbf{T}$ \\
\hline & B & 43 & $\mathbf{M}$ & Malabsorption & Nil & Nil & 58.05 & $58 \cdot 30$ & A & $3 \cdot 6$ & $3 \cdot 6$ & & & \\
\hline \multirow[b]{2}{*}{16} & $\mathbf{A}$ & 57 & $\mathbf{M}$ & Malabsorption & Nil & Nil & $65 \cdot 20$ & $65 \cdot 30$ & 0.05 & $3 \cdot 8$ & $4 \cdot 0$ & $0 \cdot 2$ & To none & $\mathbf{T}$ \\
\hline & $\mathbf{B}$ & 49 & $\mathbf{M}$ & Malabsorption & Nil & Nil & 70.55 & $70 \cdot 70$ & & 3.8 & 3.8 & A & & \\
\hline \multirow[b]{2}{*}{17} & $\mathbf{A}$ & 40 & $\mathbf{M}$ & Malabsorption & Nil & Nil & $72 \cdot 35$ & $72 \cdot 55$ & 0.05 & $4 \cdot 0$ & $4 \cdot 2$ & Nil & To none & $\mathbf{T}$ \\
\hline & B & 46 & $\mathbf{M}$ & Malabsorption & Nil & Nil & $64 \cdot 50$ & $64 \cdot 75$ & & $3 \cdot 8$ & $4 \cdot 0$ & & & \\
\hline \multirow[b]{2}{*}{18} & A & 42 & $\mathbf{F}$ & Cirrhosis of liver & Nil & Nil & 58.05 & $58 \cdot 20$ & $0 \cdot 15$ & $3 \cdot 0$ & $3 \cdot 2$ & $0 \cdot 1$ & To none & $\mathbf{T}$ \\
\hline & B & 43 & $\mathbf{M}$ & Cirrhosis of liver & Nil & Nil & 54.85 & $54 \cdot 85$ & A & $2 \cdot 7$ & $3 \cdot 0$ & B & & \\
\hline \multirow{2}{*}{19} & A & 24 & $\mathbf{M}$ & Malnutrition & Nil & Nil & 50.00 & $50 \cdot 25$ & $0 \cdot 10$ & $4 \cdot 0$ & $4 \cdot 2$ & $0 \cdot 1$ & To none & $\mathrm{T}$ \\
\hline & B & 36 & $\mathbf{M}$ & Malnutrition & Nil & Nil & $52 \cdot 50$ & 52.65 & A & $3 \cdot 7$ & 3.8 & $\mathbf{A}$ & & \\
\hline \multirow{2}{*}{20} & $\mathbf{A}$ & 40 & $\mathbf{M}$ & Malabsorption & Nil & Nil & 56.00 & 56.05 & 0.05 & $2 \cdot 3$ & $2 \cdot 2$ & $0 \cdot 2$ & To none & $\mathbf{T}$ \\
\hline & B & 48 & $\mathbf{F}$ & Malabsorption & Nil & Nil & $54 \cdot 25$ & $54 \cdot 25$ & & $2 \cdot 7$ & $2 \cdot 8$ & B & & \\
\hline
\end{tabular}

${ }^{1}$ Could not be assessed as good, moderate, or poor.

${ }^{2}$ Could not be assessed by clinical examination. The slight alteration in weight of patients was probably the result of minor changes in oedema. 
EFFECT OF INSULIN RELEASE Insulin plays an important role in the metabolism of amino-acids and protein synthesis. The plasma level of amino-acids is elevated in patients with diabetic acidosis (Luetscher, 1942). Given to normal animals (Luck and Morse, 1933) and human subjects insulin causes a fall in the blood amino-acids. Frame and Russell (1946) noted that the administration of insulin in eviscerated rats depresses the rate of increase of plasma amino nitrogen that consistently follows this operative procedure. Insulin appears to enhance the rate of penetration of amino-acids across the cell membrane, as it does the penetration of glucose, and increases protein synthesis. Krahl (1953) reported the incorporation of ${ }^{14} \mathrm{C}$-glycine into glutathione and into protein, which is greatly reduced in diabetes and is restored to normal by insulin, in liver slices. Sinex, MacMullen, and Hastings (1952), Krahl (1953), and Manchester and Young (1958) provided proof of augmentation by insulin of the incorporation of amino-acids into the protein of isolated rat diaphragm.

Pretreatment of rats with tolbutamide also increases the incorporation of ${ }^{14} \mathrm{C}$-glycine into protein in liver slices (Recant and Fischer, 1957) and when injected intravenously in human subjects decreases the serum $\alpha$-amino-acids (DeMeutter et al., 1958). It is well established that the hypoglycaemic effect of tolbutamide is mediated by a release of increased amounts of insulin from the pancreas. Since the above effects of tolbutamide are identical with those of insulin, it would appear reasonable to assume that the increase in serum proteins obtained with tolbutamide has been obtained indirectly through release of insulin by it. Probably, however, tolbutamide, in addition to what it may indirectly promote through insulin secretion, has a direct effect of its own on protein synthetis. This is indicated in animal experiments, since Mohnike, Ulrich, Bibergeil, and Czyzyk (1957) noted that after tolbutamide in pancreatectomized and alloxanized dogs, without simultaneous blood sugar lowering, the level of amino nitrogen was decreased.

One of the outstanding effects of tolbutamide therapy is weight gain. This may be yet another indirect effect through release of insulin. The anabolic effect of insulin in the absence of somatotropin is now well-established (Salter, Davidson, and Best, 1956). However, tolbutamide appears to have a protein-sparing effect of its own. Bornstein (1957) found that tolbutamide descreased gluconeogenesis from protein by inhibiting the alanine transaminase. These observations were made in vitro, and were therefore independent of insulin.

INCREASED SODIUM EXCRETION Tolbutamide, as shown by Mohnike et al. (1957), increases urinary output. The sodium excretion is also increased, as much as $40 \%$, both in patients who have been previously on insulin treatment and in those who have been not on it. This effect is, therefore, shared only by tolbutamide and may play its own part in reducing the oedema.

IMPROVED DIGESTION With tolbutamide appetite increases before the end of a week. This is probably the result of increased gastric secretion and peptic output (Weiss and Sciales, 1961) and bile flow (Kâldor and Pogátsa, 1959). Bile in turn may affect the secretion mechanism (Thomas and Crider, 1943) and promote the secretion of pancreatic juice. Tolbutamide, therefore, may promote digestion and absorption of proteins.

\section{SUMMARY}

In a double-blind trial, 20 patients with hypoproteinaemia, caused by malnutrition, malabsorption, or cirrhosis of the liver, were treated against a similar number of placebo controls. The results were assessed by sequential analysis. The criteria for assessment were subjective improvement, weight gain, reduction of oedema, and improvement in serum protein levels. A restricted closed design was chosen for the experiment. Of the 20 pairs which entered the trial, 15 pairs were in favour of tolbutamide, three pairs in favour of placebo, and two pairs were ties. The dose of tolbutamide was $0.5 \mathrm{~g}$. twice daily for four weeks. There were no adverse effects and the drug was well tolerated. The beneficial effect of tolbutamide is discussed.

We are indebted to Dr. F. J. Mendonca, Dean, B.J. Medical College, Poona, for permission to use the hospital records; and to Mr. G. Winternitz of Hoechst Pharmaceuticals for the supplies of Rastinon (tolbutamide) and placebo tablets.

\section{REFERENCES}

Armitage, P. (1960). Sequential medical trials, pp. 37 and 50. Blackwell Scientific Publications, Oxford.

Bhattacharyya, A. K., and Mandal, J. N. (1963). Tolbutamide in cirrhosis of the liver. Bull. Calcutta Sch. trop. Med., 11, 65-66.

Bornstein, J. (1957). Inhibition of alanine transaminase by the hypoglycaemic sulphonylurea derivatives. Nature (Lond.), 179, 534-535.

DeMeutter, R. C., Khachadurian, A. K., and Marble, A. (1958). Immediate effects of intravenous injections of tolbutamide and insulin on blood glucose and amino acids. Proc. Soc. exp. Biol. (N.Y.), 99, 33-35.

Dobrzański, T. (1959). The influence of tolbutamide (D860) on the liver parenchyma in diabetes mellitus. Germ. med. Mth., 4, 229-230.

Frame, E. G., and Russell, J. A. (1946). The effects of insulin and anterior pituitary extract on the blood amino nitrogen in eviscerated rats Endocrinology, 39, 420-429. 
Harrison, G. A. (1957). Chemical Methods in Clinical Medicine, pp. 408-410. Churchill, London.

Káldor, A., and Pogátsa, G. (1959). Effect of tolbutamide on bile secretion. Lancet, 2, 1094.

Knick, B., and Emrich, K. (1957). Reversibilität von pathologischem Serum-Labilitätsproben, Elektrophoresediagramm und Leberfunktionstests bei chronischen Lebererkrankungen (chronische Hepatitis, Lebercirrhosen) unter Sulfonylharnstoffgaben (D 860). Klin. Wschr., 3, 812-814.

Krahl, M. E. (1953). Incorporation of $C^{14}$-amino acids into glutathione and protein fractions of normal and diabetic rat tissues. J. biol. Chem., 200, 99-109.

László, B., Bruckner, P., Görgey, E., and Tóth, B. (1959). Acut hepatitises betegek kezelése peroralis antidiabeticummal (Bucarban), Orv. Hetil., 100, 1411-1413.

Luck, J. M., and Morse, S. W. (1933). The effects of insulin and adrenaline on the amino-acid content of blood, Biochem. J., 27, 1648-1654.

Luetscher, J. A., Jr. (1942). Metabolism of amino acids in diabetes mellitus. J. clin. Invest., 21, 275-279.

Manchester, K. L., and Young, F. G. (1958). The effect of insulin on incorporation of amino acids into protein of normal rat diaphragm in vitro. Biochem. J., 70, 353-358.
Mohnike, G., Ulrich, H., Bibergeil, H., and Czyzyk, A. (1957). Beobachtungen während der Einstellung von Diabetikern auf N-(4-Methyl-benzolsulfonyl)-N'-butyl-harnstoff (D 860). Dtsch. med. Wschr., 82, 1526-1528.

Pote, W. W. H., Jr., Anderson, E. A., and Cochran, B., Jr. (1959). Long-term clinical and laboratory studies in diabetic patients treated with tolbutamide. A preliminary report. In Diabetes Mellitus, III Kongress der international Diabetes Federation, 1958. pp. 419-425. Thieme, Stuttgart.

Recant, L., and Fischer, G. L. (1957). Studies on the mechanism of tolbutamide hypoglycemia in animal and human subjects. Ann. N.Y. Acad. Sci., 71, 62-70.

Salter, J. M., Davidson, I. W. F., and Best, C. H. (1956). Insuline, hormone de croissance. Brux. med., 36, 2377-2384.

Sinex, F. M., MacMullen, J., and Hastings, A. B. (1952). The effect of insulin on the incorporation of $\mathrm{C}^{14}$ into the protein of rat diaphragm. J. biol. Chem., 198, 615-619.

Singh, I., Sehra, K. B., and Bhargava, S. P. (1961). Tolbutamide in cirrhosis of the liver. Lancet, 1, 1144-1146.

Thomas, J. E., and Crider, J. O. (1943). The effect of bile in the intestine on the secretion of pancreatic juice. Amer. J. Physiol., 138, 548-552.

Weiss, A., and Sciales, W. J. (1961). The effect of tolbutamide on human basal gastric secretion. Ann. intern. Med., 55, 406-415.

\section{The April 1965 Issue}

\section{THE APRIL 1965 ISSUE CONTAINS THE FOLLOWING PAPERS}

Motility of the pelvic colon

Part IV Abdominal pain associated with colonic hypermotility after meals A. M. CONNELL, F. AVERY JONES, and E. N. ROWLANDS

Effect of different foods on the acidity of the gastric contents in patients with duodenal ulcer

Part I A comparison between two 'therapeutic' diets and freely-chosen meals J. E. LENNARD-JONES and N. BABOURIS Part II Effect of varying the size and frequency of meals N. BABOURIS, JANET FLETCHER, and J. E. LENNARD-JONES

Observations from New South Wales on the changing incidence of gastric ulcer in Australia B. P. BILLINGTON

Relationship between pre-operative bleeding and perforation and bleeding after operations for duodenal ulcer M. J. S. LANGMAN

Radiological appearances in achlorhydria B. A. SCOBIE and G. P. G. SIM

The pancreas and iron absorption A. E. DAVIS and J. C. BIGGS

Measurement of trypsin and chymotrypsin activity in stools to detect chronic pancreatic disease J. G. BANWELL, P. J. LEONARD, and RITA M. F. LOBO
A case of unexplained gastrointestinal protein loss R. D. H. STEWART and J. H. STEWART

A review of 436 cases of intestinal obstruction in Ibadan G. J. COLE

The pressure profile of the gastroduodenal junctional zone in dogs. BENNO M. BRINK, JERRY F. SCHLEGEL, and CHARLES F. CODE

Serum complement levels in active ulcerative colitis J. FLETCHER

Familial ulcerative colitis PETER J. MORRIS

Peutz-Jeghers syndrome with metastasizing duodenal carcinoma J. P. WILLIAMS and ANTHONY KNUDSEN

Surface carcinoma of the stomach M. K. MASON

Correlation between pathological and clinical findings in 195 consecutive liver biopsies showing brown pigment NIELS TYGSTRUP, TORBEN SCHIøDT, and KJELD WINKLER

Effect of thyroxine on the isolated rat intestine A. J. MATTY and B. SESHADRI

British Society of Gastroenterology

Copies are still available and may be obtained from the PUBLISHING MANAGER, BRITISH MEDICAL ASSOCIATION, TAVISTOCK SQUARE, W.C.I, price 18s. 6D. 